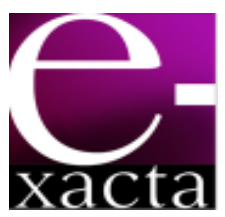

ISSN: 1984-3151

\title{
APLICAÇÃO DE MORINGA OLEIFERA LAM NO TRATAMENTO DE EFLUENTE PROVENIENTE DA LAVAGEM DE JEANS
}

\author{
MORINGA OLEIFERA LAM APPLICATION IN THE TREATMENT OF \\ EFFLUENT FROM THE WASH JEANS
}

\author{
Ana Paula Ströher'; Osorio Moreira Couto Junior²;Maraísa Lopes de Menezes ${ }^{3}$; \\ Rosângela Bergamasco ${ }^{4}$; Nehemias Curvelo Pereira ${ }^{5}$
}

1 Mestre em Engenharia Química. Universidade Estadual de Maringá, 2007. Doutoranda em Engenharia Química PEQ/UEM. Maringá, PR. ana stroher@hotmail.com

2 Mestre em Engenharia Química. Universidade Estadual de Maringá, 2008. Doutorando em Engenharia Química PEQ/UEM. Maringá, PR. osorio eq@yahoo.com.br

3 Mestre em Engenharia Química. Universidade Estadual de Maringá, 2007. Doutoranda em Engenharia Química PEQ/UEM. Maringá, PR. maraisalm@hotmail.com

4 Doutora em Engenharia Química. Universidade Estadual de Maringá, 1984. Professora da Universidade Estadual de Maringá, PR. leiladeniseff@hotmail.com

5 Doutor em Engenharia Química. Universidade Federal de Sergipe, 1970. Professor da Universidade Estadual de Maringá, PR. nehemias@deq.uem.br

Recebido em: 15/06/2012 - Aprovado em: 30/06/2012 - Disponibilizado em: 30/07/2012

\begin{abstract}
RESUMO: Nos últimos anos, a indústria nacional interessou-se em desenvolver coagulantes naturais para tratamento de efluentes e investir efetivamente na pesquisa destes novos produtos biodegradáveis de origem vegetal. Nesse contexto, estudos vêm mostrando que sementes de Moringa oleifera Lam são eficientes para a clarificação de água e efluentes. Assim, o presente trabalho teve como objetivo verificar as condições operacionais ótimas para maximizar os percentuais de remoção de cor e turbidez de um efluente proveniente da lavagem de jeans, em relação à atuação do coagulante natural, moringa, bem como a caracterização deste efluente antes e após o tratamento de coagulação-floculação. Na tentativa de realizar a otimização do processo, variou-se os tempos de mistura rápida (2 e 5 minutos), tempo de mistura lenta ( 20 e 30 minutos), e tempo de sedimentação (20 e 30 minutos), com agitação fixada em $95 \mathrm{rpm}$ para coagulação e $30 \mathrm{rpm}$ para a floculação, e as concentrações adicionadas do coagulante natural foram $1400 \mathrm{mg} / \mathrm{L}, 1600 \mathrm{mg} / \mathrm{L}, 1800 \mathrm{mg} /, 2000 \mathrm{mg} / \mathrm{L}, 2200 \mathrm{mg} / \mathrm{L}$, $2400 \mathrm{mg} / \mathrm{L}$ e $2600 \mathrm{mg} / \mathrm{L}$. Após os ensaios de coagulação/floculação em Jar Test, os parâmetros cor e turbidez foram analisados e apresentaram remoções superiores a $80,33 \%$ e $91,10 \%$, respectivamente.
\end{abstract}

PALAVRAS-CHAVE: Moringa oleifera Lam. Efluente Têxtil. Coagulação.

ABSTRACT: In recent years, the national industry was interested in developing natural coagulants for wastewater treatment and invest effectively in the research of these new biodegradable products of plant origin. In this context, studies have shown that seeds of Moringa oleifera Lam are efficient for the clarification of water and wastewater. Thus, this study verifies the optimum operating conditions that maximized the percentage of removal of color and turbidity of the effluent to study regarding the performance of natural coagulant, Moringa, as well as the characterization of after treatment of coagulation-flocculation. In an attempt to perform the optimization process, was varied rapid mixing times (2 and 5 minutes), time of slow mixing (20 and 30 minutes), and settling time (20 and 30 minutes), with agitation set at $95 \mathrm{rpm}$ to $30 \mathrm{rpm}$ for coagulation and flocculation. The additional concentrations of natural coagulant were $1400 \mathrm{mg} / \mathrm{l}, 1600 \mathrm{mg} / \mathrm{l}, 1800 \mathrm{mg} / 2000 \mathrm{mg} / \mathrm{l}, 2200 \mathrm{mg} / \mathrm{l}, 2400 \mathrm{mg} / \mathrm{L}$ and $2600 \mathrm{mg}$ / $L$ to find the optimal concentration to be added. After the tests of coagulation / flocculation in Jar Test, the 
parameters color and turbidity removals were analyzed and presented in excess of $80.33 \%$ and $91.10 \%$ respectively.

KEYWORDS: Moringa oleifera Lam. Textile Effluent. Coagulation.

\section{INTRODUÇÃo}

No Brasil, a indústria têxtil se destaca entre os oito setores mais importantes da atividade industrial, ocupando os primeiros lugares em empregos diretos $\mathrm{e}$ em faturamento. Embora as diferenças tecnológicas entre as pequenas e grandes indústrias sejam bastante significativas, todas têm em comum a particularidade de utilizar grandes quantidades de água. Este fato, associado ao baixo aproveitamento dos insumos (corantes, detergentes, engomantes, amaciantes, etc.), faz com que a indústria têxtil seja responsável pela geração de grandes volumes de resíduos, com elevada carga orgânica e forte coloração (PERALTA-ZAMORA, 2005).

Os despejos de lavanderias industriais são normalmente tratados por meio de processo físicoquímico composto coagulação/floculação/sedimentação.

O principal agente coagulante utilizado nas indústrias é o Sulfato de Alumínio. Este composto é adicionado sem um critério pré-determinado e muitas vezes em excesso, ocorrendo aumento da matéria orgânica e custos do processo (BRAILE; CAVALCANTI, 1993).

O mecanismo de coagulação/floculação provocado pela proteína existente na polpa da Moringa oleifera Lam assemelha-se ao mecanismo provocado pelos polieletrólitos (DAVINO, 1976).

A Moringa, como é mais conhecida no Brasil, a espécie Moringa oleifera Lam, é uma planta originária da Índia, que já se espalhou por todo mundo, principalmente nos países tropicais. A sua propagação pode ser feita através de sementes, mudas ou estacas. É uma planta de múltiplos usos. Na alimentação humana em alguns países são aproveitadas: as folhas como verduras cruas, as vagens verdes como verduras cozidas e as sementes maduras podem ser torradas para fabricação de farinha. As sementes também produzem um excelente óleo que pode ser usado na alimentação e para fazer sabão e cosméticos. Suas flores são muito procuradas pelas abelhas. A Moringa ainda pode ser utilizada como cerca viva e quebra ventos (GERDES, 1996).

O presente trabalho teve como objetivo propõe avaliar a eficiência do coagulante natural Moringa oleifera Lam na remoção na remoção de cor e turbidez em função da alteração dos tempos de misturas e sedimentação, a fim de se obter as condições de operação ideais para $\circ$ tratamento do efluente proveniente da lavagem de jeans.

\section{METODOLOGIA}

\subsection{COLETA DO EFLUENTE}

O efluente utilizado foi coletado no tanque de equalização de uma lavanderia industrial de jeans, localizada em Astorga-PR.

Após a coleta o efluente foi caracterizado e armazenado em um freezer a $4^{\circ} \mathrm{C}$.

\subsection{CARACTERIZAÇÃO DO EFLUENTE}

Para caracterizar o efluente, foram determinados os parâmetros cor e turbidez antes e após o tratamento proposto. Estas análises foram realizadas segundo procedimento recomendado pelo Standard Methods (APHA, 1995). 


\subsection{PREPARAÇÃO DO COAGULANTE}

Para a preparação do coagulante em pó as sementes foram descascadas e secas por seis horas em estufa (Digimec, CLTV), à $70^{\circ} \mathrm{C}$. Finalizada essa etapa, as sementes secas foram trituras em moinho (Tecnal, TE 345,60 ciclos) até formarem um pó fino e então foram peneiradas para seleção de grânulos $(0,5 \mathrm{~mm})$. O pó resultante desse processo, como mostra a Figura 1 , foi utilizado nos ensaios em Jar Test.

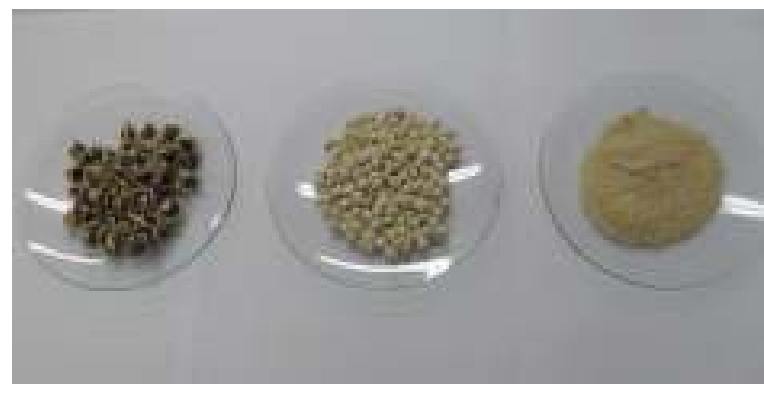

Figura 1: Sementes de moringa com casca, sem casca e em pó.

\subsection{EXPERIMENTOS DE}

\section{COAGULAÇÃO/FLOCULAÇÃO}

Os ensaios de coagulação/floculação foram realizados em Jar Test simples, Milan, Modelo JT 101/6 de seis provas, com regulador de rotação das hastes misturadoras.

Utilizou-se béqueres contendo $500 \mathrm{~mL}$ de efluente bruto e em cada um desses, foram adicionadas quantidades pré-determinadas do coagulante Moringa oleifera Lam.

A velocidade utilizada no Jar Test para propiciar a mistura rápida foi fixada em $95 \mathrm{rpm}$ e a velocidade utilizada para a mistura lenta foi $35 \mathrm{rpm}$. Esses valores foram baseados em valores já utilizados pela indústria. As concentrações adicionadas do coagulante Moringa oleifera Lam foram 1400 mg/L 1600 mg/L, 1800 mg/L,
$2000 \mathrm{mg} / \mathrm{L}, 2200 \mathrm{mg} / \mathrm{L}, 2400 \mathrm{mg} / \mathrm{L}$ e $2600 \mathrm{mg} / \mathrm{L}$ e variou-se o tempo de mistura rápida, tempo de floculação e sedimentação de forma a determinas as melhores condições para o processo, conforme a Tabela 1.

Tabela 1: Variação do tempo de mistura rápida, tempo de mistura lenta e sedimentação.

\begin{tabular}{|c|c|c|c|}
\hline Ensaio & $\begin{array}{c}\text { TMR } \\
(\mathbf{m i n})\end{array}$ & $\begin{array}{c}\text { TML } \\
(\mathbf{m i n})\end{array}$ & $\begin{array}{c}\text { SED } \\
(\mathbf{m i n})\end{array}$ \\
\hline 1 & 5 & 30 & 30 \\
\hline 2 & 5 & 20 & 30 \\
\hline 3 & 5 & 20 & 20 \\
\hline 4 & 5 & 30 & 20 \\
\hline 5 & 2 & 30 & 30 \\
\hline 6 & 2 & 20 & 30 \\
\hline 7 & 2 & 20 & 20 \\
\hline 8 & 2 & 30 & 20 \\
\hline
\end{tabular}

Após cada ensaio coletou-se o sobrenadante da amostra de cada béquer e realizaram-se as análises de cor e turbidez a fim de verificar a eficiência do tratamento em estudo.

\section{RESULTADOS E DISCUSSÃO}

Com os dados obtidos nos ensaios referentes à Tabela 1, construiu-se as superfícies de respostas para os parâmetros cor e turbidez com o auxílio do programa Statistica ${ }^{\circledR} 6.0$.

As Figuras 2 e 3 mostram a superfície de resposta e sua respectiva superfície de contorno para o parâmetro cor. 
Cor $=65,3546+39,1739^{*} x+0,1377^{*} y-5,4303^{*} x^{*} x-0,0001^{*} x^{*} y-3,0711 E-5^{*} y^{*} y$

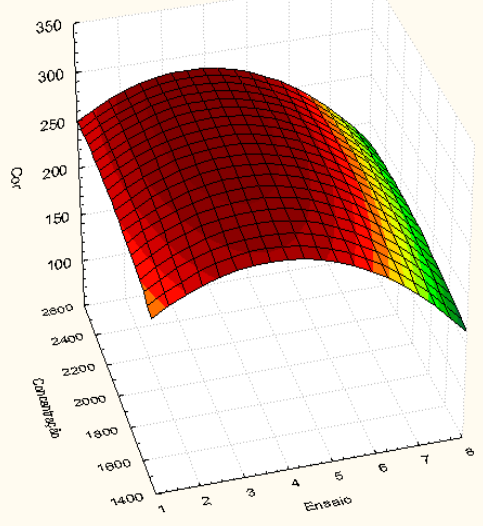

Figura 2: Superfície de resposta referente ao parâmetro cor e sua variação de acordo com o ensaio e a concentração do coagulante moringa.

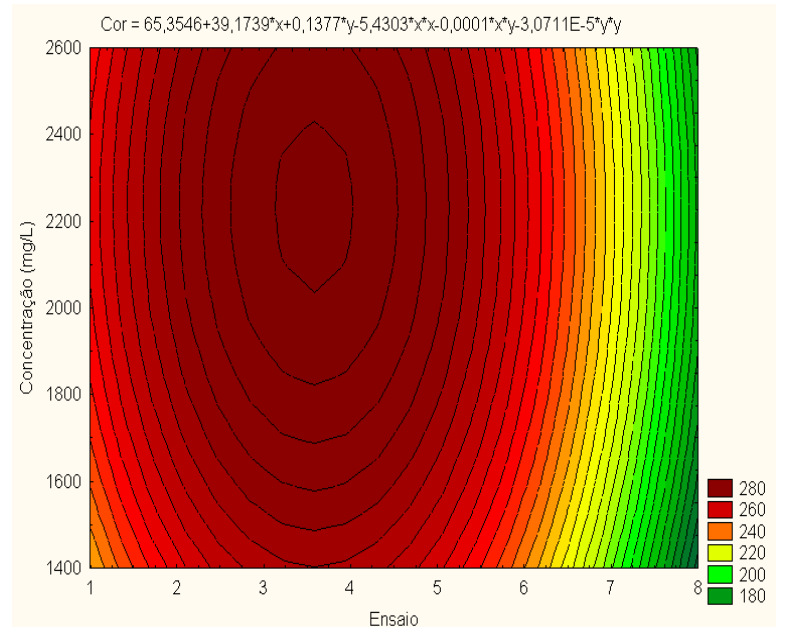

Figura 3: Contorno da superfície de resposta referente ao parâmetro cor e sua variação de acordo com o ensaio e a concentração do coagulante moringa.

A Tabela 2 apresenta os valores da análise de variância para os fatos e para a interação, de acordo com o parâmetro cor em estudo.

Tabela 2: Análise de variância para os fatores e para a interação (parâmetro cor)

\begin{tabular}{|c|c|c|c|}
\hline $\begin{array}{c}\text { Fonte de } \\
\text { Variação }\end{array}$ & $\begin{array}{c}\text { Graus de } \\
\text { Liberdade }\end{array}$ & $\begin{array}{c}\text { Soma dos } \\
\text { Quadrados }\end{array}$ & -valor \\
\hline $\mathrm{X}_{\mathrm{E}}$ & 1 & 19510,1 & 0,000610 \\
\hline $\mathrm{X}_{\mathrm{C}}$ & 1 & 2457,07 & 0,212681 \\
\hline $\mathrm{X}_{\mathrm{E}^{*}} \mathrm{X}_{\mathrm{E}}$ & 1 & 69355,44 & 0,000000 \\
\hline $\mathrm{X}_{\mathrm{C}^{*}} \mathrm{X}_{\mathrm{C}}$ & 1 & 2028,13 & 0,257232 \\
\hline $\mathrm{X}_{\mathrm{E}^{*}} \mathrm{X}_{\mathrm{C}}$ & 1 & 1,56 & 0,974887 \\
\hline $\begin{array}{c}\text { Erro } \\
\text { Total }\end{array}$ & 106 & 1563,06 & \\
\hline
\end{tabular}

Como pode-se observar nas Figura 2 e 3, e de acordo com o p-valor apresentado na Tabela 2 , a remoção de cor nesse caso, teve maior significância de acordo com o ensaio, independente da concentração de coagulante adicionado. Desse modo, para o ensaio 8 foi possível se obter a maior remoção de cor, sendo possível um valor inferior a 200 units Pt Co. Para otimizar o tratamento, conclui-se então que os melhores resultados foram obtidos para o ensaio 8 utilizando a menor concentração de moringa (1400 $\mathrm{mg} / \mathrm{L})$.

A superfície de resposta correspondente ao parâmetro turbidez e sua superfície de contorno, seguem nas figuras 4 e 5 . 
Turbidez $=54,1454+10,3053^{*} x+0,0228^{*} y-2,1977^{*} x^{*} x+0,0021^{*} x^{*} y-7,4219 E-6^{*} y^{*} y$

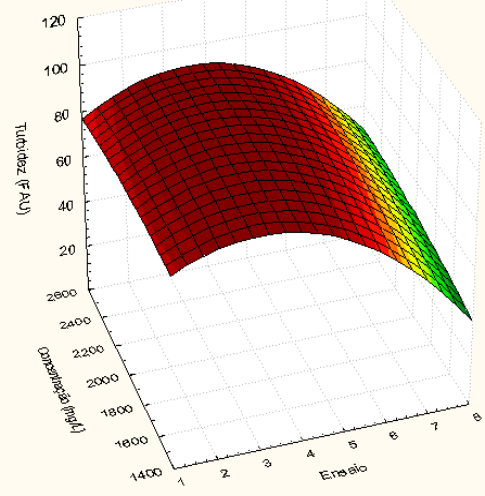

Figura 4: Superfície de resposta referente ao parâmetro turbidez e sua variação de acordo com o ensaio e a concentração do coagulante moringa.

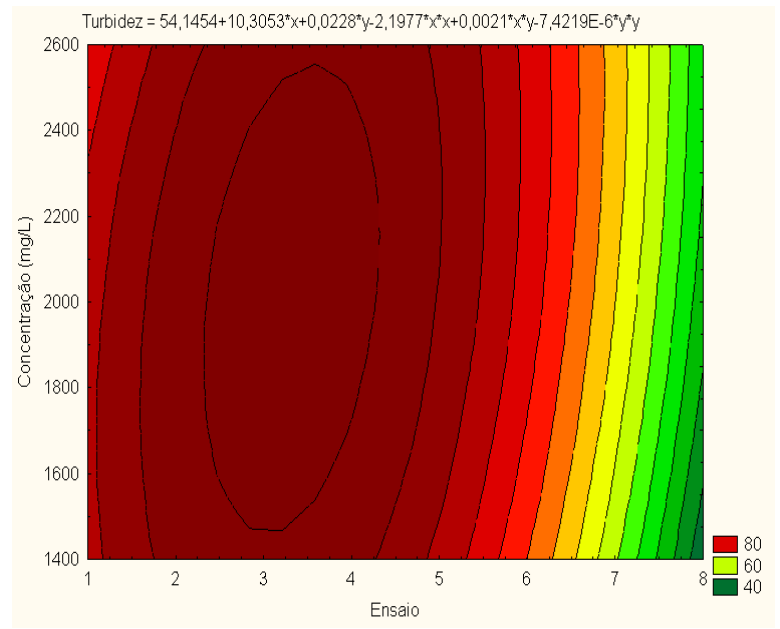

Figura 5: Contorno da superfície de resposta referente ao parâmetro turbidez e sua variação de acordo com o ensaio e a concentração do coagulante moringa.

A Tabela 3 apresenta os valores da análise de variância para os fatos e para a interação, de acordo com o parâmetro turbidez em estudo.
Tabela 3: Análise de variância para os fatores e para a interação (parâmetro turbidez)

\begin{tabular}{|c|c|c|c|}
\hline $\begin{array}{c}\text { Fonte de } \\
\text { Variação }\end{array}$ & $\begin{array}{c}\text { Graus de } \\
\text { Liberdade }\end{array}$ & $\begin{array}{c}\text { Soma dos } \\
\text { Quadrados }\end{array}$ & $\mathrm{p}$-valor \\
\hline $\mathrm{X}_{\mathrm{E}}$ & 1 & 1350,2 & 0,354665 \\
\hline $\mathrm{X}_{\mathrm{C}}$ & 1 & 67,4 & 0,835866 \\
\hline $\mathrm{X}_{\mathrm{E}^{*}} \mathrm{X}_{\mathrm{E}}$ & 1 & 11359,9 & 0,008150 \\
\hline $\mathrm{X}_{\mathrm{C}^{*}} \mathrm{X}_{\mathrm{C}}$ & 1 & 118,5 & 0,783576 \\
\hline $\mathrm{X}_{\mathrm{E}^{*}} \times \mathrm{X}_{\mathrm{C}}$ & 1 & 420,5 & 0,604971 \\
\hline
\end{tabular}

Analisando as Figura 4 e 5, e de acordo com o p-valor apresentado na Tabela 3, a remoção de turbidez do efluente, novamente, teve maior significância de acordo com o ensaio, independente da concentração de moringa. Assim, para o ensaio 8 foi possível se obter a maior remoção turbidez, resultando um valor inferior a $40 \mathrm{FAU}$. Otimizando o tratamento, conclui-se então que os melhores resultados foram obtidos para o ensaio 8 utilizando a menor concentração de moringa (1400 mg/L).

A Tabela 5 apresenta os parâmetros de cor, turbidez e DQO para o efluente bruto e para o efluente tratado com sementes de moringa nas condições ótimas determinadas no ensaio.

Tabela 5: Parâmetros cor, turbidez e DQO antes e após o tratamento com sementes de moringa, submetido às condições ótimas determinadas no ensaio.

\begin{tabular}{|c|c|c|}
\hline Parâmetro & $\begin{array}{c}\text { Efluente } \\
\text { Bruto }\end{array}$ & $\begin{array}{c}\text { Efluente } \\
\text { Tratado }\end{array}$ \\
\hline $\begin{array}{c}\text { Cor } \\
\text { (units Pt Co) }\end{array}$ & 1017 & 200 \\
\hline $\begin{array}{c}\text { Turbidez } \\
\text { (FAU) }\end{array}$ & 449 & 40 \\
\hline
\end{tabular}




\section{NOMENCLATURA}

TMR (min): tempo de mistura rápida

TML (min): tempo de mistura lenta

SED (min): tempo de sedimentação

\section{CONCLUSÕES}

A conclusão deste trabalho é que para o tratamento de um efluente de lavanderia de jeans, o coagulante Moringa oleifera Lam mostrou-se uma boa opção alternativa, pois apresentou remoções satisfatórias dos parâmetros estudados.

O melhor percentual de remoção dos parâmetros estudados foi obtido utilizando-se a menor concentração de coagulante, correspondente a 1400 $\mathrm{mg} / \mathrm{L}$. Em relação aos tempos de mistura, o ensaio ótimo foi 0 de número 8 , com tempos de mistura rápida, lenta e sedimentação igual a 2, 30 e $20 \mathrm{~min}$, respectivamente.

Comparando o efluente bruto e o efluente tratado com moringa percebe-se remoções superiores a $80,33 \%$ para o parâmetro cor, $91,10 \%$ para turbidez.

\section{NOMENCLATURA}

TMR (min): tempo de mistura rápida

TML (min): tempo de mistura lenta

SED (min): tempo de sedimentação

\section{REFERÊNCIAS}

APHA - American Public Health Association. "Standard Methods for the Examination for Water and Wastewater". 19 ${ }^{\text {th }}$ ed., AWWA, WPCF, Washington, D.C.; 1995.

BRAILE, P. M.; CAVALCANTI, J.E.W.A. Manual de Tratamento de Águas Residuárias, São Paulo: CETESB, 1993, 0764.

DAVINO, F. "Tecnologia de tratamento de água: água na indústria”. Rio de Janeiro: Almeida Neves, 1976.
GERDES, G. "Uso das sementes da árvore Moringa oleifera para tratamento de águas turvas". Fortaleza: ESPLAR, 1996.

PERALTA-ZAMORA, P.; SOUZA, L. R. C. Degradação de corantes reativos pelo sistema ferro metálico/peróxido de hidrogênio. Química Nova, 208: 226-228, 2005. 